\title{
Flux Calibration Issues
}

\author{
Andrew J. Pickles \\ Caltech, 1200 E. California Blvd., Pasadena, CA 91225, USA \\ email: pick@astro.caltech.edu
}

\begin{abstract}
Recent stellar spectral libraries have sought higher resolution and the accurate determination of specific optical spectral indeces as stellar population indicators. But the value of accurate flux comparisons over wide wavelength regions should still be emphasized, particularly as more and better spectro-photometric data for composite populations becomes available.
\end{abstract}

\section{Introduction}

Several new stellar spectral libraries have emerged in the last few years, offering broad coverage of stellar temperature, gravity and abundance. They achieve good spectral resolution of about 2000-5000 or more, and are generally observed with fairly narrow apertures. This paper seeks to emphasize the value of time spent ensuring accurate flux calibration of library spectra, the value to potential users of providing good error estimates, and of accurately describing the instrumentation and methodologies used.

\section{Spectral Libraries}

Table 1 lists some libraries that have been published since 2000, together with their resolution, wavelength coverage, number of spectra, temperature and metallicity ranges, aperture sizes used, and estimated overall flux calibration accuracies.

Spectra in the STELIB library (Le Borgne et al. 2003) were observed through a 1.5" slit at the 1-m JKT on La Palma and through a 2" slit at the 2.3-m ANU telescope. They have been checked and compared with extensive UBV and RI photometric data from the Lausanne GCPD photometric database (Mermilliod, Mermilliod \& Hauck 1997) to achieve a photometric accuracy of about $3 \%$ overall. The library has been used to construct some widely used stellar population models (Bruzual \& Charlot 2003), although improvements in some of the wavelength calibrations, and in the flux calibrations may be possible.

The INDO-US library (Valdes et al. 2004) was observed through a 1.4" slit at the Coudé feed of the KPNO 2.1-m telescope. PCA fitting and spectral energy distributions (SEDs) have been used to interpolate some gaps in the spectra. The data have been used for automated spectral classification, and provide a large database of high-resolution spectra over a wide wavelength range. Its utility will be further improved with improvements to the flux calibration, possibly by cross checks and calibration against other data sets.

The ELODIE library and continuing extensions and improvements (Prugniel \& Soubiran 2001, Prugniel \& Soubiran 2004) was observed through 2" aperture fibers on the OHP 1.9-m telescope. The spectra have generally been calibrated to better than $2.5 \%$ accuracy via extensive internal and external comparisons. It is used with the PEGASE spectral synthesis system (Le Borgne et al. 2004).

The MILES library (Sánchez-Blázquez et al. 2006, Cenarro et al. 2007) was observed on the Intermediate Dispersion Spectrograph of the 2.5-m INT (La Palma) through a 0.7 " slit, with additional exposures through a wide slit to ensure accurate flux calibration. 
Table 1. Recent Stellar Spectral Libraries

\begin{tabular}{|c|c|c|c|c|c|c|c|c|}
\hline $\begin{array}{l}\text { Library } \\
\text { (Ref) }\end{array}$ & $\begin{array}{l}\text { Telescope/ } \\
\text { Instrument }\end{array}$ & $\begin{array}{l}\text { Resolution/ } \\
\text { elmt/pix }(\AA)\end{array}$ & $\begin{array}{l}\text { Wavelength } \\
\text { Range (nm) }\end{array}$ & $\begin{array}{c}\mathrm{N} \\
\text { Spectra }\end{array}$ & $\begin{array}{c}\mathrm{T}_{e f f} \\
(' 000 \mathrm{~K})\end{array}$ & $\begin{array}{l}{[\mathrm{Fe} / \mathrm{H}]} \\
\text { Range }\end{array}$ & $\begin{array}{c}\text { Apertures } \\
\text { (") }\end{array}$ & $\begin{array}{c}\text { Flux } \\
\text { Accuracy }\end{array}$ \\
\hline STELIB $^{1}$ & $\mathrm{JKT} / \mathrm{ANU} 2.3 \mathrm{~m}$ & $2000 / \sim 3 / 1$ & $320-930$ & 250 & $4-32$ & $-1, .15$ & $1.5 / 2$ & $3 \%$ \\
\hline INDO-US $^{2}$ & KPNO $2.1 \mathrm{~m}$ & $5000 / \sim 1 / 0.4$ & $346-946$ & 1270 & $3-32$ & $-3, \quad .5$ & 1.4 & $\sim 5 \%$ \\
\hline ELODIE $^{3}$ & OHP $1.9 \mathrm{~m}$ & $42000 / \sim .3 / .05$ & $400-680$ & 4000 & $3-60$ & $-3.2, \quad .4$ & 2 & $<\sim 2.5$ \\
\hline MILES $^{4}$ & INT $2.5 \mathrm{~m}$ & $2000 / 2.3 / 0.9$ & $352-750$ & 1000 & $3-40$ & $-2.7, \quad 1.0$ & $0.7 / 6$ & $1.3-2.5$ \\
\hline UVES POP 5 & VLT & $80000 / .1 /$ & $307-1030$ & 350 & & & 0.5 & high \\
\hline $\mathrm{NGSL}^{6}$ & HST/STIS & $>1000 / 5 / 1.4$ & $170-1025$ & 380 & $3-60$ & $-2.5, \quad .5$ & 0.2 & $1-1.5$ \\
\hline $\mathrm{NGSL}^{7}$ & IRTF/SpEX & $2000 / \sim 4 / \sim 2$ & $800-2500$ & 100 & & & $0.3 / 3$ & high \\
\hline LW $2000^{8}$ & ANU $2.3 \mathrm{~m}$ & $1100 / \sim 40 / \sim 10$ & $970-2490$ & 140 & $3-20$ & $-1, \quad 0.0$ & $1 / 10$ & good \\
\hline
\end{tabular}

References:

(1) Le Borgne et. al., 2003; (2) Valdes et. al., 2004; (3) Prugniel \& Subiran, 2001, 2004

(4) Sánchez-Blázquez et. al. 2006; (5) Bagnuolo et. al., 2003; (6) Gregg et. al., 2004

(7) http://irtfweb.ifa.hawaii.edu/ spex/spexlibrary/IRTFlibrary.html

(8) Lançon \& Wood, 2000

Extensive comparisons with other data indicate that this has been achieved to the $1.5 \%$ to $2.5 \%$ level. This library has the largest metallicity coverage, including spectra of some super metal-rich stars.

The UVES Paranal Observatory Project library (Bagnuolo et al. 2003) is interesting in that it comprises serendipitous spectra taken with the VLT UVES instrument, mainly during twilight calibration procedures. The data are high resolution, high $\mathrm{S} / \mathrm{N}$ and high quality. The data taken through a narrow 0.5 " slit are not absolutely flux calibrated, but provide a good relative flux calibration with standard ESO extraction and calibration procedures.

The New Generation Spectral Library (NGSL, Greg et al. 2004) provides HST/STIS spectra of about 400 stars from the uv to 1 micron. The data require custom processing because of the non-standard observational technique used, and some early data have required additional calibration. Because they are taken in space and are unaffected by telluric absorption or atmospheric or seeing variations, when fully published these data should provide the best flux calibration of recent libraries, although the resolution is lower. This library has been extended to the infrared for about 100 spectra with the cross dispersed infrared SpEX spectrograph (Rayner et al. 2003) on the 3-m IRTF, with observations taken through a 0.3 " slit, and a 3" slit for flux calibration.

A library of cool stars was also observed by Lançon \& Wood (2000) using the CASPIR cross dispersed spectrograph on the ANU $2.3 \mathrm{~m}$. The spectra were calibrated against Kurucz model atmospheres and internal reference stars to provide transmission functions for the instrument and for the atmosphere, which can vary on short timescales. More infrared program and spectral library data should emerge from the coordinated NIRES (Keck), TripleSpec (Hale) and Virginia (APO) instrument development, eg. http://www . astro.virginia.edu/research/instrumentation/cormass 2 .php

\section{Flux Calibration methods}

Flux calibration methods are almost as diverse in practice as there are practitioners, but all good methods rely on careful attention to detail, and a number of direct and indirect comparisons between internal and external data.

A good understanding of the instrumental effects is necessary to enable proper removal of the instrumental signature: calibrate the detector variations (flat-fielding), extract the spectra, subtract the background and sky signals, and provide wavelength calibration. 
Variable instrumental throughput due to flexure, degradation of optics or sensors, or changes in instrumental setup must also be understood and calibrated to ensure proper flux calibration.

There are many optical standards, including Hamuy et al. (1992, 1994). Cohen and his group (eg. Cohen et al. 1999) established a large set of infrared standards across the sky, tied to Kurucz stellar atmosphere models. They also corrected errors introduced eg. during previous flux calibrations of the Low Resolution Spectrograph on the IRAS satellite when Aldebaran was assumed to be a black body, which had had the effect of making the $\mathrm{SiO}$ absorption in the Aldebaran spectrum appear as false emission features in other spectra.

For near infrared spectra Vacca, Cushing \& Rayner (2003) present a method to remove telluric absorption based on comparison with A0 star spectra. Cushing, Vacca \& Rayner (2004) provide further details about spectral extraction and calibration.

In most cases, and assuming stable equipment, varying atmospheric effects dominate the resulting accuracy of the flux calibration, both for overal SED and removal of telluric features. The effect of telluric features can be alleviated by division by a suitable atmospheric standard observed close in time and in the same way. The atmospheric standard must be chosen with care for the wavelength region of interest. Removal of telluric absorption will not change the reduced $\mathrm{S} / \mathrm{N}$ in the features: a companion spectrum showing the variable error as a function of wavelength is particularly useful for anyone seeking to use these libraries, and critical when attempting to combine spectra of varying accuracy.

Seeing variations cause differential light loss with wavelength, even for slits aligned along the parallactic angle. As observatory seeing monitors clearly show (eg. http:// www.not.iac.es/weather/getData.php?s=robodimm, and http://odata1.palomar. caltech.edu/massdimm), seeing varies on short time scales, so the flux correction curve appropriate to light though a narrow slit may vary quite quickly. If spectra cannot be observed through a wide aperture to provide a comparison SED, the flux-calibrated, narrow-aperture spectrum can still be checked for continuum fit against a suitable synthetic spectrum (e.g. Gustaffson, Martins, Coelho in this volume, Kurucz SYNTHE code, Munari et al. 2005), or against known filter photometry. Such external checks provide a realistic validation of the overall flux calibration accuracy.

\section{Filters}

Bessell (2005) provides an extensive review of photometric standard systems and synthetic photometry comparisons. He describes the techniques and difficulties of synthetically reproducing some older photometric systems, and raises the issue that some colors such as the Johnson $U$ band may no longer be accurately reproducible. But Maíz Apellániz (2006) restores faith in this process by computing a new U band filter transmission that provides accurate reproducibility for a wide range of colors. It is important obviously to use accurate filter transmission curves when comparing synthetic with observed photometry, and to clearly describe the systems used.

Checks against broad band photometry can best be done by comparing synthetic photometry of flux calibrated spectra against the object's known photometric colors. It is often simpler however to overlay and check fluxes derived from photometric colors against partially flux calibrated spectra. The latter should obviously be done at the effective wavelength of the filter for the type and color of star under consideration. The broader wings of the Johnson/Cousins filters may permit an error in one part of the spectrum to appear to manifest itself in another. Sloan SDSS filters provide somewhat better band 
definitions, their effective wavelengths change less with stellar color, and the amount of comparison data continues to increase.

For all libraries, the increasing amount and availability of photometric data enables and encourages additional checks and corrections. For example a check of synthetic SDSS photometry of the HILIB library (Pickles 1998) against SDSS colors of standard types shows linear fits with dispersions of $0.027,0.026,0.030$ and 0.072 magnitudes for g-r, r-i, $\mathrm{r}-\mathrm{z}$ and $\mathrm{u}-\mathrm{g}$ respectively, within the expected combined errors.

\section{Conclusions}

Modern spectral libraries provide a significant increase in the availability of spectral data for an increasingly wide range of stellar parameters. These provide the basis for the accurate analysis of composite populations, through the developing dataset from new telescopes and instruments. Several authors have demonstrated the ability to maintain accurate flux calibration even at high resolution. These checks and an accurate assessment of residual errors are time consuming and laborious, but are clearly necessary to ensure the continued utility of these libraries for analysis of composite populations.

\section{References}

Bagnuolo, S., Jehin, E., Ledoux, C., Cabanac, R., Melo, C. \& Gilmozzi, R. 2003, ESO Messenger 114,10

Bessell, M. S. 2005, Ann. Rev. 43, 293

Bruzual, G. \& Charlot, S. 2003, MNRAS 344, 1000

Cenarro, A.J., Peletier, R.F., Sánchez-Blázquez, P, Selam, S.O., Toloba, E., Cardiel, N., FalcónBarroso, J, Gorgas, J., Jiménez-Vicente, J. \& Vazdekis, A. 2007, MNRAS 374, 664

Cohen, M., Walker, R. G., Carter, B, Hammersley P., Kidger M. \& Noguchi, K. 1999, AJ, 117, 1864

Cushing, M. C., Vacca, W. D. \& Rayner, J. T. 2004, PASP 116, 362

Gregg, M. D., Silva, D., Rayner, J., Valdes, F., Worthey, G., Pickles, A., Rose, J. A., Vacca, W. \& Carney, B. 2004, BAAS 36, 1496

Hamuy M., Walker A. R., Suntzeff N. B., Gigoux P., Heathcote S. R. \& Phillips M. M. 1992 PASP 104, 533

Hamuy M., Suntzeff N. B., Heathcote S. R., Walker A. R., Gigoux P. \& Phillips M. M. 1994 PASP 106, 566

Lançon, A. \& Wood, P. R. 2000, A\&AS 146, 217

Le Borgne, J.-F., Bruzual, G., Pelló, R., Lançon, A., Rocca-Volmerange, B., Sanahuja, B., Schaerer, D., Soubiran, C. \& Vílchez-Gómez, R. 2003, A\&A 402, 433L

Le Borgne, D., Rocca-Volmerange, B., Prugniel, P., Lançon, A., Fioc, M. \& Soubiran C. 2004, A\&A 425,881

Maíz Apellániz, J. 2006, ApJ 131, 1184

Mermilliod, J.-C., Mermilliod, M. \& Hauck, B. 1997, A\&AS 124, 349

Munari, U., Sordo, R., Castelli, F. \& Zwitter, T. 2005 A\&A 442, 1127

Pickles, A. J. 1998, PASP 110, 863

Prugniel, Ph., \& Soubiran, C. 2001, A\&A 369, 1048

Prugniel, Ph., \& Soubiran, C. 2004, astro.ph 0409214

Rayner, J. T, Toomey, D. W., Onaka, P. M., Denault, A. J., Stahlberger, W. E., Vacca, W. D., Cushing, M. C. \& Wang, S. 2003, PASP 115, 362

Sánchez-Blázquez, P., Peletier, R.F., Jiménez-Vicente, J., Cardiel, N., Cenarro, A.J., FalcónBarroso, J., Gorgas, J., Selam, S. \& Vazdekis, A. 2006, MNRAS 371, 703

Vacca, W. D., Cushing, M. C. \& Rayner, J. T 2003, PASP 115, 389

Valdes, F., Gupta, R., Rose, J. A., Singh, H. P. \& Bell D. J. 2004, ApJS 152, 251 


\section{Discussion}

Gustafsson: Just a plea to those publishing libraries of observed spectra, corrected by SEDs from theoretical models - document (and even published) exactly what you do since we shall certainly change the theoretical models.

PICKLES: I agree - just as updates and corrections to theoretical spectra are described in publications.

HeAP: The NGSL has flux calibration issues. Around a quarter of the program stars were off-center in the 0.2 arcsec slit, and so are affected by slit vignetting at long wavelengths $(\lambda>\mathrm{V}$ band $)$.

PICKLES: a) Compare several spectra of known type to their corresponding theoretical spectra and derive corrections curves to bring their continua into correspondence. Is there a single common curve that could be applied to all NGSL spectra to remove the 'bubble'?. b) Acquire accurate girz colors for all NGSL stars and apply to fit spectra.

MEIXNER: With all these wonderful new IR spectrographs coming on line, is there anyone coordinatin an effort to complement the wonderful optical spectral libraries that are in place?.

PICKLES: Although ESO has managed to do this it has (apparently) impacted other science programs. The science initiatie needs to come from scientific collaborations, but each proposal (to each TAC) has to make sense and win time on its own merit.

TRAGER: There will be no GAIA filter set: it will output two low resolution spectra with $\mathrm{R} \sim 20-80$ in the blue and $\mathrm{R} \sim 65-100$ in the red. This is due to a complete redesign of the instrument using two prisms rather than filters.

PICKLES: That is a real shame, but will photometry derived from prism spectra still be possible?. As Bessell (and others) have shown, the SDSS set can be modified (e.g. U) to improve its sensitivity to astrophysical parameters. Also, UBVRI data is convertible to ugriz. Separable filters should be better for comparing photometric and spectroscopic data, and comparisons with theoretical results. 